\title{
Design of A Method of Resisting Random Broadband Electromagnetic Interference based on Cognitive Radio and Its Application
}

\author{
Naji Ma ${ }^{1,2, a}$, Yi Wang ${ }^{3}$, Zhikai Wang ${ }^{4}$, Jikun Guo ${ }^{2}$, Fugang $\mathrm{Liu}^{2}$ and Feng $\mathrm{Xu}^{2}$ \\ ${ }^{1}$ College of Information and Communication Engineering, Harbin Engineering University, Harbin \\ 150001, China; \\ ${ }^{2}$ Information and Network Center, Heilongjiang University of Science and Technology, Harbin \\ 150022, China; \\ ${ }^{3}$ Heilongjiang Province Institute of Supervision and Inspection of Electronic Products, Harbin \\ 150001, China; \\ ${ }^{4}$ College of Shipbuilding Engineering, Harbin Engineering University, Harbin 150001, China. \\ a5203datashare@163.com
}

\begin{abstract}
Keywords: communication in coal mine, electromagnetic interference, convergence optimization, cognitive radio, fruit fly optimization algorithm.
\end{abstract}

\begin{abstract}
There exist many kinds of electromagnetic interference sources in a small and enclosing underground coal mine work area, which could lead to that the instable phenomenon of the wireless monitoring and communication systems constantly occur. Focused on the randomness of occurrence and duration of electromagnetic interference and the diversity of interference frequency band in underground coal mines, a kind of anti-electromagnetic method based on full duplex(FD) cognitive radio was presented. In present work, the transmission parameters such as transmission power and carrier frequency at each communication node $(\mathrm{CN})$ can be easily adjusted by detecting the surrounding electromagnetic environment at correspondent CNs. When the frequency band being used by CNs is disturbed, the communication task can be kept by switching to other available frequency bands. By doing this, the whole network can be reached the approximation of optimal communication status. The simulation results show that the present method can obtain higher network efficiency by effectively avoiding frequency bands that are subjected to electromagnetic interference or cause data transmission conflicts. Furthermore, the anti-interference ability and the spectrum utilization efficiency of wireless communication systems in underground coal mines are obviously improved.
\end{abstract}

\section{Introduction}

The environment of wireless communication in the coal mine underground is inadequate. High-power electrical equipment rebooted frequently, novel electronic spares and the radio frequency equipment widely being applied generate a large number of electromagnetic interference which has leaded the performance of coal mine wireless communication systems especially wireless emergency protection systems were unstable. The situation has seriously endangered the safety of coal mine production [1].

A solution to the problem of electromagnetic interference in coal mines is to eliminate or reduce interference from the interference sources. In [2], a method of that connecting a shielded conductor with a zero-signal reference potential at the signal source ground ensure that the parasitic current flows only in the shield was proposed to suppress by the external electromagnetic interference. In [3], according to the characteristics of the transient surge radiation generated during start-up and shutdown of the pump motor, the corresponding shielding measures are proposed to reduce the interference to the data transmission. However, with the coal mine automation and information technology improved, the number of interference sources is also increased. In practical applications, the effect was not satisfied by using of a single shielding method with a large of work quantity. Another solution of the interference was to improve the anti-jamming capability of wireless 
communication systems. Based on the fixed carrier parameter transmission mode commonly used in traditional mine communication, in [5] an anti-jamming method was proposed based on the combined the power of location with channel adjustment to improve the transmitting power of the $\mathrm{CN}$. The objective of the method improves the communication performance by high energy consumption. However, because there exist mine gas, coal dust and other flammable gases in the coal mine, it requires high level of electrical explosion proof. Taking into account the safety of production, the provisions of the transmit power cannot exceed 25W [6]. In addition, the excessive transmit power will shorten the work cycle of the CNs in the coal mine which are mostly battery-powered. Thus, the issues of $\mathrm{CN}$ energy-saving need to be considered in the engineering application.

However, comparing with the ground, the spectrum in the coal mine belongs to an open source that can be fully utilized [4]. When the carrier signals using different frequency and power are transmitted in the coal mine, the impact level of interference which was caused by the length and shape of tunnel, metal support and large electromechanical equipment is quite different. Based on the characteristics of the mine communication environment, FD cognitive radio theory is introduced into the mine wireless communication system. All the uninterrupted frequency bands in the network are regarded as open shared resources. The spectrum resources are concentrated and allocated by using fruit fly algorithm (FOA) to achieve dynamic adjusting the transmission power, carrier frequency, coding for each CN. When the signal-to-interference-plus-noise ratio (SINR) of a frequency band that is being used by the $\mathrm{CN}$ is lower than the specified tolerance threshold, the CN can be immediately switched to other available frequency bands continuing the communication task. This method can significantly improve the anti-jamming ability and spectrum utilization efficiency of the wireless communication system in coal mine, and it can also make full use of the frequency bands which are not used due to the interference before.

\section{The Frequency Characteristic Analysis of Interference Source in Coal Mine}

Although the electromagnetic interference in coal mine is in line with the general law electromagnetic interference, but because of its particularity electromagnetic environment and equipment, there are unique laws from the type of interference to the coupling of transmission channels [9]. In the paper, the formation mechanism, frequency characteristics of major interference sources and its influence on wireless communication in the coal mine are discussed by analyzing the electromagnetic interference data collected in BiShan coal mine in[9], QianYingZi coal mine in[2], and TaShan coal mine in[3] and the following three conclusions can be obtained:

(1)There are a variety of sources of interference in coal mine and many of them are mobile interference sources. The interfered frequency band is narrow and widely distributes in a wide frequency range.

(2)These interferers have a strong randomness to the time and duration of interference.

(3)The electromagnetic attenuation rate of the interference source in coal mine is higher. The interference coverage is limited only to its nearby $\mathrm{CN}$ to generate high energy local interference [6]. The interfering band, field strength and other indicators of several major interference sources in coal mine was listed in Table 1.

Therefore the situation of the interference for each $\mathrm{CN}$ in coal mine is different. 
Table 1. The disturbing characteristics of interference sources in coal mine

\begin{tabular}{cccc}
\hline $\begin{array}{c}\text { Type of interference } \\
\text { source }\end{array}$ & $\begin{array}{c}\text { Interfered frequency } \\
\text { band/ }(\mathrm{MHz})\end{array}$ & $\begin{array}{c}\text { Max interfered } \\
\text { frequency/ }(\mathrm{MHz})\end{array}$ & $\begin{array}{c}\text { Interference field } \\
\text { strength/ }\left(\mathrm{dBmV} \cdot \mathrm{m}^{-1}\right)\end{array}$ \\
\hline Frequency converter & $0 \sim 1200$ & 119 & -31.5 \\
Power grid & $0.5 \sim 4$ & 3 & -26.3 \\
Lighting system & $0.1 \sim 3$ & 1 & -57.9 \\
Electric locomotive & $100 \sim 160$ & 138 & -34.5 \\
Feed switch & $30 \sim 200$ & 75 & -10.5 \\
Pump & $0.14 \sim 1$ & 0.766 & 0.121 \\
Substation & $0 \sim 180$ & 112 & -19.9 \\
\hline
\end{tabular}

\section{Design of Anti-Electromagnetic Noise Jamming Method based on FD Cognitive Radio}

Based on the characteristics of coal mine environment and electromagnetic noise, the working principle of the proposed anti-jamming method is shown in Fig 1.

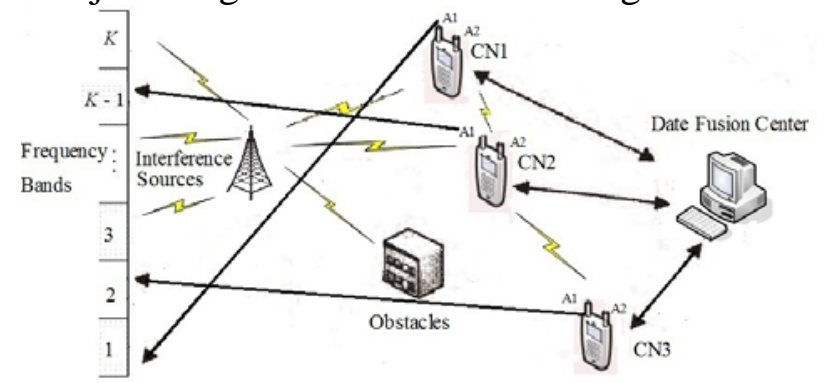

Fig. 1 The anti-interference communication system of full duplex cognitive radio

As we can see that each $\mathrm{CN}$ is equipped with two antennas. In each slot, the A-antenna is used to sense the external communication environment of the CN and the B-antenna is used to transmit data. The character of this kind of real-time sensing technology by using independent antenna is no blind spots detection. The $\mathrm{CN}$ uses the energy detection method to sense the external electromagnetic environment, and then uploads the sensing result to the data fusion center. First, the data fusion center processes all detection results by using pre-defined fusion methods, and then establishes an open shared resource model (spectrum common model) in the system to share all unperturbed spectrums (channels) for the users. The algorithm is used to optimize the shared resource model, in order to maximize the spectrum utilization [10]. Each CN adjusts the transmission parameters according to the assigned channel, effectively to improve the anti-interference ability of the $\mathrm{CN}$, and to ensure that the communication collision between the cognitive users does not occur. In addition, once the SINR of the channel falls below the specified tolerance threshold, the CN can immediately exit and search other available channels to avoid interference from electromagnetic noise.

\subsection{The Strategy of Spectrum Sensing}

A cooperative energy spectrum sensing method using energy detection is applied in the method. The energy detection block diagram is shown in Figure 2.

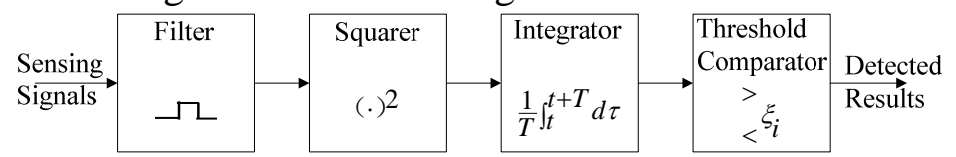

Fig. 2 The block diagram of the energy detector

The observational statistic of the ${ }^{i} \mathrm{CN}$ in the system can be expressed as

$$
e_{i}=\frac{1}{N} \sum_{n=1}^{N}\left|x_{i}(k)\right|^{2}
$$

Where $N$ is the number of sampling points. When the power of the signal detected in the channel is higher than the preset threshold value, the channel is deemed as not suitable for data transmission, otherwise the channel can be used. The $d_{i}$ is used to represent the detection result of the $i \mathrm{CN}$.

$$
d_{i}= \begin{cases}0, & e_{i} \leq \xi_{i} \\ 1, & e_{i}>\xi_{i}\end{cases}
$$


Where the $\xi_{i}$ represents the threshold value of signal detection. The performance of spectrum detection can be described by the false alarm probability and detection probability under the condition of certain SNR. When there is only additive Gaussian noise in the channel, the detected signal's statistics follow the central chi-square distribution. When the channel has been mixed strong electromagnetic noise or other CNs are using the channel to send the data, the statistics are subject to non-central chi-square distribution. From the central limit theorem, both the chi-squared distribution and the non-chi-square distribution can be approximately regarded as Gaussian distribution when the number of sampling points is enough. When the detection threshold was given, the false alarm probability $P_{f(i)}$ and detection probability $P_{d(i)}$ can be expressed as:

$$
\begin{aligned}
& P_{f(i)}=P\left(e_{i}>\xi_{i} \mid H_{0}\right)=Q\left(\frac{(N / 2)^{1 / 2} \cdot\left(\xi_{i}-\sigma_{i}^{2}\right)}{\sigma_{i}^{2}}\right) \\
& P_{d(i)}=P\left(e_{i}>\xi_{i} \mid H_{1}\right)=Q\left(\frac{(N / 2)^{1 / 2} \cdot\left(\xi_{i}-\left(1+\chi_{i}\right) \cdot \sigma_{i}^{2}\right)}{\left(1+2 \chi_{i}\right)^{1 / 2} \cdot \sigma_{i}^{2}}\right)
\end{aligned}
$$

Where the signal-to-noise ratio of the received signal is denoted $\chi_{i}$ as the $i \mathrm{CN}$. $Q(\cdot)$ can be expressed as

$$
Q(x)=\frac{1}{\sqrt{2 \pi}} \int_{x}^{+\infty} \exp \left(-\frac{u^{2}}{2}\right) \mathrm{d} u
$$

After the $\mathrm{CN}$ detects the channel is detected by the $\mathrm{CN}$, the consequences will be uploaded to the data fusion center. The detection results of each CN which is represented by $D=\left[d_{1, \ldots,} d_{i}, \cdots, d_{M}\right]$ are fused by the data fusion center according to the OR logic merge rule. With the number of users increased, the probability of detection also increases. The false alarm probability $Q_{f}$ and detection probability $Q_{d}$ of the system can be expressed as:

$$
\begin{gathered}
Q_{f}=1-\prod_{i=1}^{M}\left(1-P_{f(i)}\right) \\
Q_{d}=1-\prod_{i=1}^{M}\left(1-P_{d(i)}\right)
\end{gathered}
$$

\subsection{Channel Allocation Strategy of Eliminating Interference}

The anti-jamming channel assignment strategy belongs to the discrete combinatorial optimization problem, which is a NP problem [11]. In this paper, a relatively new optimization algorithm of FOA is used to solve the problem [12].

The population of fruit flies was randomly divided into $p$ parallel subpopulations. Each of subpopulations is composed of $l$ fruit flies, which is equal to the number of undisturbed channels. The search precision of the algorithm is determined by the value of $p$, and the calculating of construction engineering quantity and convergence precision should be taken into account, when setting its quantity. In order to ensure the global nature of the search, the initial coordinate positions of all fruit flies are represented by a two-dimensional array of random values within the domain.

A food source taste concentration comparison $P=\left\{p_{i, j} \mid p_{i, j} \in\{0,1\}\right\}_{p \times l}$ matrix which is used to update the channel allocation scheme. The updating rule can be denoted:

$$
p_{i, j}=\left\{\begin{array}{l}
1, \text { if } \varepsilon_{i j}^{t}>\varepsilon_{i}^{\text {best }} \\
0, \text { if } \varepsilon_{i j}^{t} \leq \varepsilon_{i}^{\text {best }}
\end{array}\right.
$$

Where $\varepsilon_{i j}{ }^{t}$ the food taste concentration is value of the $j$ fruit fly in the sub-population after $t$ iteration; $\varepsilon_{i}^{\text {best }}$ is the historical optimum concentration value of the sub-population $i$.

The best spectrum allocation policy by the $N \times M$ matrix $\Psi=\left\{\psi_{n, m} \mid \psi_{n, m} \in\{0,1\}\right\}_{N \times M}$, where $\psi_{n, m}=1$ means that channel is assigned to theCN $n$ otherwise $\psi_{n, m}=0$. 


\section{Simulation Result Evaluation and Analysis}

The simulation experiments are assumed that the main types of interference sources. Its number, and interference radiation radius in the mine fully mechanized mining work area are set as shown in Table 2.Since the displacement of the mobile interference source in a sensing time slot is very short, it is assumed that the locations of the interference sources are fixed for each sensing slot.

Table 2. The disturbing characteristics of interference sources in coal mine

\begin{tabular}{ccc}
\hline $\begin{array}{c}\text { Type of interference } \\
\text { sources }\end{array}$ & $\begin{array}{c}\text { The number of } \\
\text { interference sources }\end{array}$ & $\begin{array}{c}\text { The range of each } \\
\text { interference source }\end{array}$ \\
\hline Mining equipment & 1 & 3.5 (in meters) \\
Electromechanical & 1 & 4.0 (in meters) \\
transmission equipment & 2 & 2.5 (in meters) \\
VVVF equipment & 2 & 6.0 (in meters) \\
KBZ & 1 & 4.5 (in meters) \\
Mobile substation & 1
\end{tabular}

Furthermore, it is assumed that the number of CNs in the system is 7, and 5 orthogonal channels are available. In order to validate the effectiveness of the proposed method, the cooperative spectrum sensing experiments are carried out in the SNR environment of $-5 \mathrm{~dB},-8 \mathrm{~dB},-10 \mathrm{~dB}$ and the number of CNs with 3, 5, 7 respectively. The simulation results are shown in Figure 3.

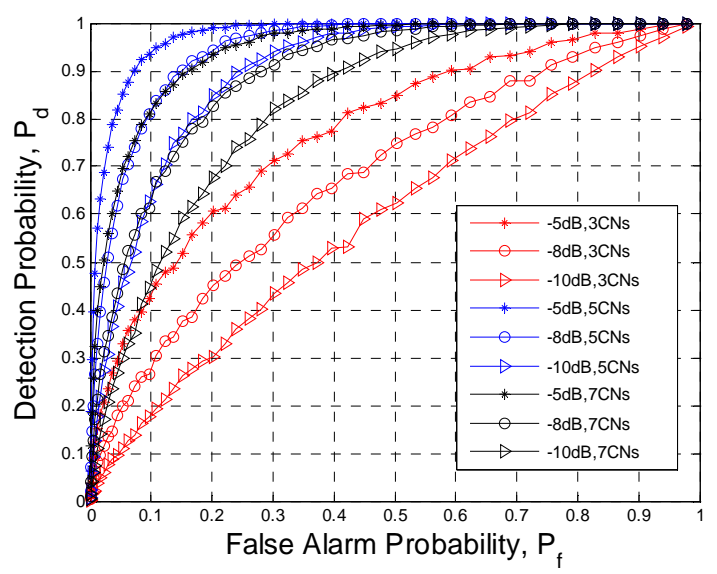

Fig. 3 The performance of spectrum detection by using the proposed method

The simulation results in Figure 4 show the effectiveness of the proposed method. Especially in the case of $7 \mathrm{CNs}$, even if the signal-to-noise ratio is $-10 \mathrm{~dB}$, higher detection accuracy can be obtained.

The number of fruit fly population is defined as $p=20$, the stopping criterion is 500 . All experimental results are averaged over 40 run simulations. In order to verify the effectiveness of the method, two classical PSO and GA algorithms are also used to solve the problem. The experimental results are compared with the method. Without loss of generality, the scale of PSO and GA is set to 20 and the number of iterations is also 500. The simulation curves of the three algorithms based on the maximum network efficiency function and the maximum proportion fairness network benefit function are shown in Fig.4 and Fig.5.

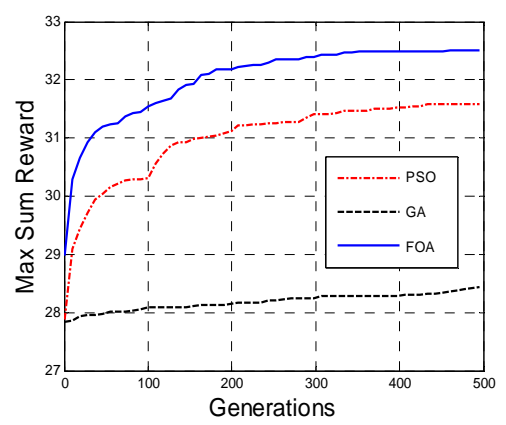

Fig. 4 Comparison of stimulating results of MSR

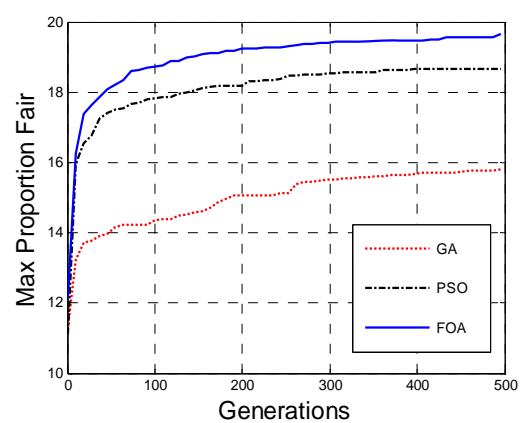

Fig. 5 Comparison of simulating results of MPF 
From the simulation results, we can see that the network benefits obtained by the system under different objective functions are improved during the iterative process. This shows that the CN can dynamically adjust the transmission channel to select the optimal channel according to the change of the network environment and the proposed method is verified. In addition, the convergence speed of GA is too fast and easily falls into local convergence at the initial iteration. Compared with the GA and the PSO, the FOA got a better global convergence and higher network efficiency.

\section{Conclusion}

A kind of electromagnetic interference jamming method in the coal mine underground using cognitive radio is proposed in the paper. All undisturbed spectrums obtained by CN's real-time monitoring are regarded as open shared resources, and the FOA algorithm is used to allocate them among the CNs to achieve the target of anti-electromagnetic noise interference and improving the spectrum utilization efficiency. In the proposed method, the fruit fly population was divided into several subpopulations, which could effectively improve the efficiency of searching optimal solution. Experimental results show that the $\mathrm{CN}$ can dynamically adjust the transmission parameters according to the detected results avoiding the electromagnetic interference. The proposed method achieved maximum efficiency of network with a reward of 32.61 after 500 iterations. The anti-interference ability and spectrum utilization efficiency have been effectively improved.

\section{Acknowledgements}

I would like to thank the supporting of Natural Science Foundation of China (No.51474100) and Natural Science Foundation of Heilongjiang Province of China (No.QC2016093) for the paper.

\section{References}

[1]. Tian Jie, Wang Hongrao, et al. Research on the coupling of electromagnetic interference in the monitoring system of coal mine wire rope. Journal of China Coal Society. Vol. 41(2016) No. 4, p. $1054 \sim 1058$.

[2]. Liao Zhiqiang, Chen Dongchum, Liu Shuiwen. Research of underground electromagnetic interference sources and anti-interference technology. Industry and Mine Automation. Vol. 7(2012) No. 7, p.25 28.

[3]. Sun Jiping, Zheng Zhaowen, Feng Dewang, et al. Influence of surge on electromagnetic environment in the mine pump house. Journal of China Coal Society. Vol. 35(2010) No. 2, p.349 352.

[4]. Tang Liang, Zhang Shen, Gan Ling, et al. Application research of cognitive radio technology in coal mine underground. Industry and Mine Automation. Vol. 7(2010) No. 7, p.36 39.

[5]. Li Lifen, Yu Yonghua, Zhu Yongli. Analysis of wireless sensor network for transmission line monitoring. high voltage engineering. Vol. 37(2011) No. 5, p.1180 1185.

[6]. Sun Jiping. Research on characteristics and key technology in coal mine Internet of things. Journal of China Coal Society. Vol. 36(2011) No.1, p.167 171.

[7]. Chen Guizhen, Ding Enjie, Zhang Shen, et al. Brief analysis of application of cognitive radio in mine communication. Industry and Mine Automation. Vol. 6(2009) No. 6, p.12 14.

[8]. Pan W T. A new fruit fly optimization algorithm: taking the financial distress model as an example. Knowledge-Based Systems. Knowledge Based Systems. Vol. 26(2012) , p.69 74.

[9]. Zou Zheqiang, Zhang Jie. Measurement and analysis of underground electromagnetic interference of medium and low frequency band. Industry and Mine Automation. Vol. 39(2013) No.5, p.1 5.

[10]. Peng Chunyi, Zheng Haitao. Utilization and fairness in spectrum assignment for opportunistic spectrum access. ACM Mobile Networks and Applications. Vol. 11(2006) No.4, p.555 576. 
[11]. Gao Hongyuan. Quantum-inspired bee colony optimization algorithm and its application for cognitive radio spectrum allocation. Journal of Central South University. Vol. 43(2012) No.12, p.4743 4749.

[12]. Wang Xuegang, Zuo Zaojian. FOA-Based SVM parameter optimization and its application in ship manoeuvring prediction. Journal of Shanghai Jiao Tong University. Vol. 47(2013) No.6, p.884 888. 